American Journal of Applied Sciences 7 (6): 835-839, 2010

ISSN 1546-9239

(C) 2010Science Publications

\title{
Synthesis of Gold (Au) Nanoparticles for Mercury Adsorption
}

\author{
Khairul Sozana Nor Kamarudin and Mawarni Fazliana Mohamad \\ Department of Gas Engineering, Faculty of Chemical and Natural Resources Engineering, \\ University Technology Malaysia, 81310 UTM Skudai, Johor, Malaysia
}

\begin{abstract}
Problem statement: Mercury is not only hazardous to human health and the environment but could also attack equipment components resulting in a mechanical failure and gas leakage. Approach: Describes the preparation of various sizes and shapes of Au nanoparticles for mercury adsorption by using polyol method under MW heating. Au nanoparticles have been synthesized by a polyol method under Microwave (MW) heating in the presence of Au seed $\left([\mathrm{Au}]_{1} /[\mathrm{Au}]_{0}=6\right.$ of molar ratio) and different Polyvinylpyrrolidone (PVP) concentrations $(11.1,22.2$ and $33.3 \mathrm{mM}$ ). The amount of mercury adsorbed was determined by analyzing the concentration of mercury solution before and after the contacts with various sizes and shapes of Au nanoparticles. Results: When the lower PVP concentration $(11.1 \mathrm{mM})$ was used, polygonal and spherical nanoparticles were dominantly present, while spherical nanoparticles were preferentially form by using higher PVP concentrations (22.2 and $3.33 \mathrm{mM})$. It was found that the diameter and length of Au nanoparticles decreased with increasing PVP concentrations. When Au nanoparticles solution was prepared using Au seed, significant changes in sizes and shapes of Au seed occurred. Spherical nanoparticles in the Au seed $(22.2 \mathrm{mM})$ were completely changed to polygonal nanoparticles. The amount of mercury adsorbed using $9 \mathrm{ppm}$ mercury solution on polygonal nanoparticles is higher $(64.88 \%)$ than spherical nanoparticles $(18.77 \%)$. Conclusion: These results indicated that mercury adsorption strongly depends on the sizes and shapes of Au nanoparticles. Polygonal nanoparticles adsorbed more mercury than spherical nanoparticles but the amount mercury adsorbed is inversely proportional to their size.
\end{abstract}

Key words: Au nanoparticles, polyol method, microwave heating, mercury adsorption

\section{INTRODUCTION}

Mercury is present in nature and is also present in most natural gas and natural gas condensate at varying levels (Ebinghaus et al., 1999). The typical mercury concentration in natural gas and natural gas condensate is between 1 and $200 \mu \mathrm{g} \mathrm{Nm}^{-3}$ of gas (Shafawi et al., 1999). Mercury in natural gas condensate could be present in various forms (elemental, organometallic and inorganic salt), depending on the origin of the condensates.

Mercury extraction and purification of gases, as well as reworking the products of gas deposits, which involve mercury separating as a fairly toxic element, is very important for the environment protection (Spiric and Mashyanov, 2000). It is important even in cases when mercury is not an industrial component; the huge amounts of world annual reworking gas should be taken into account (Ozerova et al., 1999).
Previous study stated that the purpose of removing mercury from natural gas is to protect downstream aluminum heat exchangers in natural gas plants. Mercury has caused numerous aluminum exchanger failures. It amalgamates with aluminum, resulting in a mechanical failure and gas leakage (Wilhem and Bloom, 2000). Another reason for removing mercury is that mercury is a very volatile element. Its vapors can be a dangerous source of air pollution, thus representing a serious risk for human health (Ebinghaus et al., 1999). Exposure to high mercury levels can be harmful to the brain, heart, kidneys, lungs and immune system of humans of all ages (Darbha et al., 2007).

Among various metallic nanoparticles, the ability gold $(\mathrm{Au})$ to adsorb and amalgamate mercury is well known. Since the reactions strongly depend on sizes and shapes, the polyol method is a typical technique to prepare Au nanoparticles of different sizes and shapes by reducing their ionic salts. In general, a mixture of

Corresponding Author: Khairul Sozana Nor Kamarudin, Department of Gas Engineering,

Faculty of Chemical and Natural Resources Engineering, University Technology Malaysia,

81310 UTM Skudai, Johor, Malaysia 
reagent and polymer surfactant in Ethylene Glycol (EG) was heated in an oil bath for several hours and spherical nanoparticles were prepared. Recently Microwave (MW) heating has been coupled with the polyol method for rapid preparation of $\mathrm{Au}$ nanoparticles (Tsuji et al., 2003; 2004). This study describes the preparation of various sizes and shapes of $\mathrm{Au}$ nanoparticles for mercury adsorption by using polyol method under MW heating.

\section{MATERIALS AND METHODS}

Hydrogen tetrachloroaurate (III) hydrate $\left(\mathrm{HAuCl}_{4}\right.$. $3 \mathrm{H}_{2} \mathrm{O}$ ), is a source of Au nanoparticles, Ethylene Glycol (EG) is used as solvent and reducing agent, Polyvinylpyrrolidone (PVP) as a protecting or capping agent and Mercury Chloride $\left(\mathrm{HgCl}_{2}\right)$ is a source of mercury.

Preparation of Au nanoparticles: The MW-polyol method used in this study was similar to that reported previously (Tsuji et al., 2003; 2004). The following two procedures have been used for the preparation of $\mathrm{Au}$ nanoparticles solution. No $\mathrm{Au}$ seed are used in first procedures, while they are used in the second procedure:

- $\quad$ Procedure 1: This procedure was used for the study of fundamental effects of the different PVP concentration. $\mathrm{Au}$ nanoparticles solution were prepared by reduction of $\mathrm{HAuCl}_{4} \cdot 3 \mathrm{H}_{2} \mathrm{O}(0.02 \mathrm{~g}$ : $0.0559 \mathrm{mmol})$ in $20 \mathrm{~mL}$ ethylene glycol in the presence of various mixtures of PVP (average molecular weight: $10,000,2.22-6.66 \mathrm{~g}$ : Corresponding to $0.222-0.666 \mathrm{mmol})$. The solution was rapidly heated by MW irradiation from room temperature to the boiling point of EG $\left(198^{\circ} \mathrm{C}\right)$ for $3 \mathrm{~min}$. The product solution of PVP (4.44 g: $0.444 \mathrm{mmol}$ ) was used as Au seed.

- $\quad$ Procedure 2: This procedure was used to observe the growth of $\mathrm{Au}$ nanoparticles. Here, we define $[\mathrm{Au}]_{0}$ as a initial concentration of $\mathrm{HAuCl}_{4} \cdot 3 \mathrm{H}_{2} \mathrm{O}$ used for $\mathrm{Au}$ seed in the first step, while $[\mathrm{Au}]_{1}$ denotes a concentration of $\mathrm{HAuCl}_{4} \cdot 3 \mathrm{H}_{2} \mathrm{O}$ in the second step. Then, the $[\mathrm{Au}]_{1} /[\mathrm{Au}]_{0}$ molar ratio used in this study corresponded to 6 . The solution was heated again by MW irradiation for $3 \mathrm{~min}$

Preparation of mercury solution: The initial mercury standard solution was prepared by dissolving $0.01354 \mathrm{~g}$ of $\mathrm{HgCl}_{2}$ in $1 \mathrm{~L}$ deionized water. This solution was further diluted whenever necessary for the analysis.

Characterization of Au nanoparticles: After MW irradiation, products solutions of Au nanoparticles were centrifuged at 10,000 rpm for $2 \mathrm{~h}$. The centrifugal step was carried out twice. The precipitate was collected and dispersed on ethanol for transmission electron microscopy (JEOL JEM-2010 TEM) observation. Absorption spectra of reagent and product solutions were measured in Ultra Violet-Visible (UV-Vis) absorption spectroscopy using Jenway 6305 spectrometer. Original product solutions were diluted in ethanol by factor of 25 before the spectral measurements.

Mercury adsorption measurement: After $\mathrm{Au}$ nanoparticles solution was centrifuged, the precipitate $(0.001 \mathrm{~g})$ was added in $10 \mathrm{ml}$ mercury solution. The percentage $\mathrm{Au}$ nanoparticles to adsorb mercury were determined by analyzing the concentration of mercury solution before and after the contacts with $\mathrm{Au}$ nanoparticles. The absorbance measurements were carried out by the atomic absorption spectrophotometer. (AAS, AAnalyst 400).

\section{RESULTS}

Synthesis of Au nanoparticles: Figure 1 shows TEM photographs of Au nanoparticles prepared from procedure 1 and procedure 2. Various mixtures of spherical and polygonal nanoparticles were prepared. To obtain information on optical of $\mathrm{Au}$ nanostructures, absorption spectra of reagent and product solution in Procedures 1 and 2 were measured in UV-Vis spectra (Fig. 2).

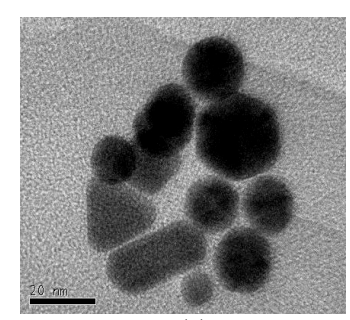

(a)

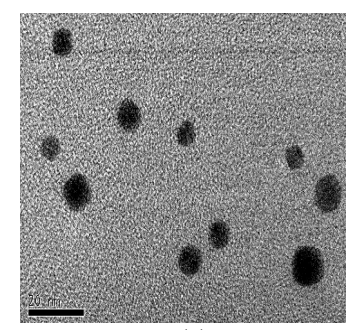

(c)

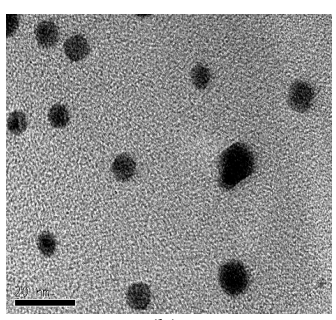

(b)

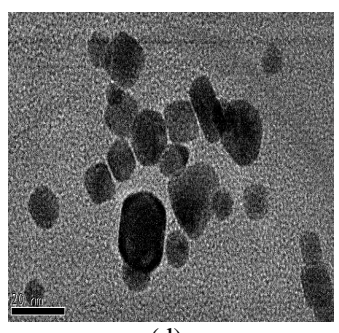

(d)
Fig. 1: TEM photographs of $\mathrm{Au}$ nanoparticles prepared from Procedure 1 (a-c) with PVP concentration (11.1-33.3 $\mathrm{mM}$ ) and (d) from Procedure 2 with $[\mathrm{Au}]_{1} /[\mathrm{Au}]_{0}$ molar ratio corresponded to 6 . (a) $11.1 \mathrm{mM}$; (b) $22.2 \mathrm{mM}$; (c) $33.3 \mathrm{mM}$; (d) $[\mathrm{Au}]_{1} /[\mathrm{Au}]_{0}=6$ 


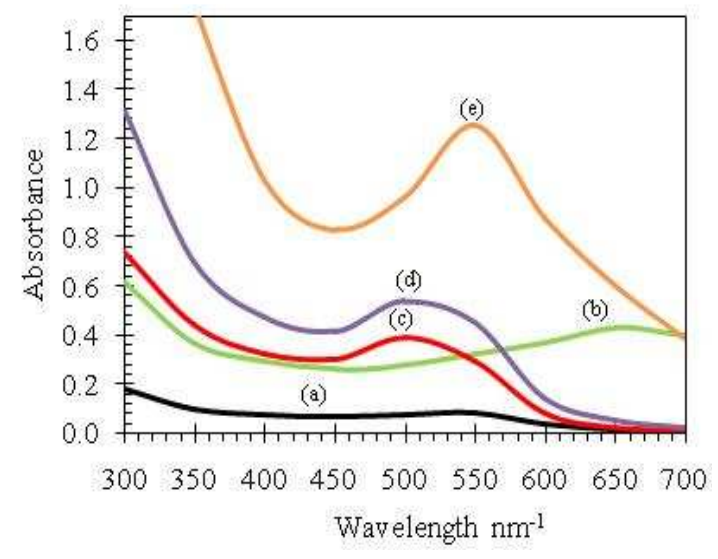

Fig. 2: Absorption spectra of original solution (a) and product solutions prepared from Procedure 1 with PVP concentration were (b) $11.1 \mathrm{mM}$, (c) $22.2 \mathrm{mM}$ (d) $33.3 \mathrm{mM}$ and (e) from Procedure (2) with $[\mathrm{Au}]_{1} /[\mathrm{Au}]_{0}$ molar ratio corresponded to 6

Table 1: AAS results of mercury adsorption mercury on $\mathrm{Au}$ nanoparticles solution

\begin{tabular}{llll}
\hline & Concentration $(\mathrm{ppm})$ & $\begin{array}{l}\text { Amount } \\
\text { mercury } \\
\text { adsorbed }\end{array}$ \\
& - Initial, & Equilibrium, & $\begin{array}{l}\text { (\%) } \\
\text { Sample }\end{array}$ \\
\hline $11.1 \mathrm{mM}$ & $\mathrm{Ce}$ & $\mathrm{Ci}$ & 54.87 \\
$22.2 \mathrm{mM}$ & 9.071 & 4.094 & 18.77 \\
$33.3 \mathrm{mM}$ & 9.071 & 7.368 & 29.38 \\
{$[\mathrm{Au}]_{1} /[\mathrm{Au}]_{0}=6$} & 9.071 & 6.406 & 64.88 \\
\hline
\end{tabular}

Mercury adsorption: Table 1 shows the concentrations of mercury solutions before and after the contacts with Au nanoparticles in order to determine the percentage of mercury adsorption on different sizes and shapes of Au nanoparticles.

\section{DISSCUSSION}

Synthesis of Au nanoparticles: Au nanoparticles were synthesized in order to examine the effects of PVP. It is known that without adding PVP, only large spherical Au nanoparticles with diameter 100-300 nm were produced in solution. These large spherical $\mathrm{Au}$ nanoparticles are formed by aggregation of small particles due to lack of surfactant PVP (Tsuji et al., 2004).

Figure 1a-c shows TEM photographs of products obtained by using Procedure 1 at the three different PVP concentration and Fig. 1d shows TEM photographs using Procedure 2 with $[\mathrm{Au}]_{1} /[\mathrm{Au}]_{0}$ molar ratio corresponded to 6 . At the lower PVP concentration (11.1 mM) larger triangular, square, hexagonal and spherical particles with diameter of $10-30 \mathrm{~nm}$ are produced (Fig. 1a). It should be note that a nanorods with a diameter of $12 \mathrm{~nm}$ and length of $30 \mathrm{~nm}$ also produced. At higher PVP concentration (22.2 mM), spherical particles with diameter 6-10 nm was produced (Fig. 1b). At the highest PVP concentration (33.3 mM), hexagonal and spherical nanoparticles with diameter 5$10 \mathrm{~nm}$ were observed (Fig. 1c). These results indicated that the diameter and length of $\mathrm{Au}$ nanoparticles decreased with increasing PVP concentrations and thus, PVP concentration plays a significant role for the size and shape of $\mathrm{Au}$ nanoparticles.

When Au seed are used in Procedure $2\left([\mathrm{Au}]_{1} /[\mathrm{Au}]_{0}\right.$ molar ratio of 6$)$, spherical nanoparticles changes to larger triangular, square and hexagonal particles with diameter of 8-20 nm. In addition, a few nanorods with diameter $8 \mathrm{~nm}$ and length of $20 \mathrm{~nm}$ was obtained. It was found that there is a growth from the $\mathrm{Au}$ seed to $\mathrm{Au}$ nanoparticles. It should be known that seeded medium synthesis is very effective for the size and shape controlled of Au nanoparticles (Jana et al., 1999; 2001; Gole and Murphy, 2004).

The formations of anisotropic Au nanoparticles start by the following reactions according to reduction mechanism of metallic ions in EG (Fievet et al., 1989):

$$
\begin{aligned}
& \mathrm{CH}_{2} \mathrm{OH}-\mathrm{CH}_{2} \mathrm{OH} \rightarrow \mathrm{CH}_{3} \mathrm{CHO}+\mathrm{H}_{2} \mathrm{O} \\
& 6 \mathrm{CH}_{3} \mathrm{CHO}+2 \mathrm{Au}^{3+} \rightarrow 2 \mathrm{Au}+6 \mathrm{H}^{+}+3 \mathrm{CH}_{3} \mathrm{COCOCH}_{3}
\end{aligned}
$$

As a result of the above reduction process, nucleation and growth processes of $\mathrm{Au}$ yield a mixture of polygonal and spherical nanoparticles. These nanoparticles were well dispersed because of the presence of a polymeric surfactant (PVP) that chemically absorb onto the surfaces of Au solid. The surface energies of large particles are lower than those of smaller one. It is therefore expected that some small nanoparticles are grown to a larger ones via an Ostwald ripening process (Roosen and Carter, 1998). With the assistance of PVP, some of the large nanoparticles were grown into anistropic rod-shaped and wire structures. In this reaction, PVP kinetically acts as a controller of the growth rates of different crystalline faces through adsorption and desorption. The PVP adsorbed on specific crystalline surfaces and could significantly decrease their growth rates and lead to highly anistropic growth (Almeida and Alcacer, 1983).

The reagent and product solution of $\mathrm{Au}$ nanoparticles in procedure 1 and procedure 2 were measured using UV-Vis spectrometer. It is known that the wavelengths and absorbance of surface plasmon bands depend on their sizes and shapes of $\mathrm{Au}$ nanoparticles. The reactant spectrum composed without 
an absorption peak. In the product spectra (Fig. 2), broad surface plasmon bands of $\mathrm{Au}$ nanoparticles appear in 500-700 nm regions. It is known that a surface plasmon band of spherical Au nanoparticles appears in the 500-600 $\mathrm{nm}$ regions with a sharp peak at about $520 \mathrm{~nm}$ (Henglein, 1999; Pastoriza-Santos and Liz-Marzan, 2002; Malikova et al., 2002) while plasmon bands of polygonal $\mathrm{Au}$ nanoparticles are observed in the 550-800 $\mathrm{nm}$ region (Tsuji et al., 2003).

Thus, strong band in the $500-600 \mathrm{~nm}$ region observed in spectra product solutions (Fig. 2c-d) are ascribed to a surface plasmon band of spherical $\mathrm{Au}$ nanoparticles while the longer wavelength bands above $600 \mathrm{~nm}$ is attributed to plasmon band of polygonal $\mathrm{Au}$ nanoparticles (Fig. 2b). The plasmon band of polygonal $\mathrm{Au}$ nanoparticles decreased with increasing the PVP concentration.

The spectrum for $[\mathrm{Au}]_{1} /[\mathrm{Au}]_{0}$ molar ratio of 6 is composed of a single peak at $550 \mathrm{~nm}$. These observation are consistent with the fact that dominant product solution of 22.2 and $33.3 \mathrm{mM}$ are spherical nanoparticles, while those product solution $11.1 \mathrm{mM}$ and $[\mathrm{Au}]_{1} /[\mathrm{Au}]_{0}$ molar ratio corresponded to 6 are polygonal nanoparticles.

Mercury adsorption performance: Equation 3 was used to determine the percentage amount of mercury adsorbed:

Percentage amount of mercury adsorbed $=\frac{C_{i}-C_{e}}{C_{e}} \times 100$

Where:

$\mathrm{C}_{\mathrm{i}}=$ Initial mercury concentration ( $\left.\mathrm{ppm}\right)$

$\mathrm{C}_{\mathrm{e}}=$ Equilibrium mercury concentration (ppm)

Table 1 summarizes the AAS result of adsorption mercury in product solution in Procedures 1 and 2. In this study, $9.071 \mathrm{ppm}$ initial concentration of mercury solution was prepared. At the low PVP concentration of $11.1 \mathrm{mM}, 54.87 \%$ mercury was adsorbed. The adsorption mercury on Au nanoparticles prepared using $22.2 \mathrm{mM}$ and $33.3 \mathrm{mM}$ was 18.77 and $29.38 \%$, respectively. At the $[\mathrm{Au}]_{1} /[\mathrm{Au}]_{0}$ of 6 , the amount of mercury adsorbed was $64.88 \%$.

The possible explanation of the adsorption is related to the sizes and shapes of Au nanoparticles. In this study, polygonal shape of Au nanoparticles adsorbed more mercury than spherical nanoparticles and the mercury adsorbed in $\mathrm{Au}$ nanoparticles is inversely proportional to their size.

\section{CONCLUSION}

In this study, MW-polyol method was applied to the rapid synthesis of $\mathrm{Au}$ nanoparticles. It was found that from TEM observation, sizes and shapes of $\mathrm{Au}$ nanoparticles strongly depend on experimental parameter such as concentration of PVP. The diameter and length of $\mathrm{Au}$ nanoparticles generally decreased with increasing the PVP concentration. The spectral observations from measurements in UV-visible absorption spectra are consistent with TEM observation of sizes and shapes of products solution. On the other hand, the growth from $\mathrm{Au}$ seed to $\mathrm{Au}$ nanoparticles changes from spherical to polygonal nanoparticles. The results show that mercury adsorption on the $\mathrm{Au}$ nanoparticles depends strongly on the sizes and shapes of Au nanoparticles. Polygonal nanoparticles adsorbed more mercury than spherical nanoparticles but the amount mercury adsorbed is inversely proportional to their size. A further study will be carried out to control the sizes and shapes of $\mathrm{Au}$ nanoparticles for optimum mercury adsorption.

\section{ACKNOWLEDGEMENT}

We kindly thank Prof M. Tsuji of Institute for Materials Chemistry and Engineering in Japan for his guidance on the preparation of $\mathrm{Au}$ nanoparticles by MW-polyol method. This research was supported by Ministry Of Higher Education (MOHE), Malaysia under Fundamental Research Grant Scheme (FRGS) and University Technology Malaysia.

\section{REFERENCES}

Almeida, M. and L. Alcacer, 1983. Growth of large single crystals of triethylammonium BIStetracyanoquinodimethane-TEA (TCNQ) $)_{2}$. J. Cryst. Growth, 62: 183-188. DOI: 10.1016/00220248(83)90023-4

Darbha, G.K., A. Ray and P.C. Ray, 2007. Gold nanoparticle-based miniaturized nanomaterial surface energy transfer probe for rapid and ultrasensitive detection of mercury in soil, water and fish. J. Am. Chem. Soc., 1: 208-214. DOI: 10.1021/nn7001954

Ebinghaus, R., R.M. Tripathi, D. Wallschlager and S.E. Lindberg, 1999. Natural and Anthropogenic Mercury Sources and their Impact on the AirSurface Exchange of Mercury on Regional and Global Scales. In: Mercury Contaminated SitesCharacterization, Risk Assessment and Remediation, Ebinghaus, R., R.R. Turner, L.D. de Lacerda, O. Vasiliev and W. Solomons (Eds.). Springer, Heidelberg, pp: 3-50. 
Fievet, F., J.P. Lagier, B. Blin, B. Beaudoin and M. Figlarz, 1989. Homogeneous and heterogeneous nucleations in the polyol process for the preparation of micron and submicron size metal particles. Solid State Ionics, 32: 198-205. DOI: 10.1016/0167-2738(89)90222-1

Gole, A. and C.J. Murphy, 2004. Seed-mediated synthesis of gold nanorods: Role of the size and nature of the seed. Chem. Mater., 16: 3633-3640. DOI: $10.1021 / \mathrm{cm} 0492336$

Henglein, A., 1999. Radiolytic preparation of ultrafine colloidal gold particles in aqueous solution: Optical spectrum, controlled growth and some chemical reactions. Langmuir, 15: 6738-6744. DOI: 10.1021/la9901579

Jana, N.R., T.K. Sau and T. Pal, 1999. Growing small silver particle as redox catalyst. J. Phys. Chem., 103: 115-121. DOI: 10.1021/jp982731f

Jana, N.R., L. Gearheart and C.J. Murphy, 2001. Wet chemical synthesis of silver nanorods and nanowires of controllable aspect ratio. Chem. Commun., 13: 617-618. DOI: 10.1039/b100521i

Malikova, N., I. Pastoriza-Santos, M. Schierhom, N.A. Kotov and L.M. Liz-Marzan, 2002. Layer-bylayer assembled mixed spherical and planar gold nanoparticles: Control of interparticle interactions. Langmuir, 18: 3694-3697.DOI: 10.1021/la025563y

Ozerova, N.A., N.P. Mashyanov, Y.I. Pikovsky, V.V. Ryzhov and A.E. Chernova et al., 1999. Mercury in Gas and Oil Deposits. In: Mercury Contaminated Sites, Ebinghaus, R., R.R. Turner, L.D. de Lacerda, O. Vasiliev and W. Solomons (Eds.). Springer, Berlin, pp: 237-246.
Pastoriza-Santos, I. and L.M. Liz-Marzan, 2002. Formation of PVP-protected metal nanoparticles in DMF. Langmuir, 18: 2888-2894. DOI: 10.1021/la015578g

Roosen, A.R. and W.C. Carter, 1998. Simulations of microstructural evolution: Anisotropic growth and coarsening. Physica, 261: 232-247. DOI: 10.1016/S0378-4371(98)00377-X

Shafawi, A., L. Ebdon, M. Foulkes, P. Stockwell and W. Corns, 1999. Determination of total mercury in hydrocarbons and natural gas condensate by atomic fluorescence spectrometry. Analyst, 124: 788-796. DOI: $10.1039 / \mathrm{a} 809679 \mathrm{a}$

Spiric, Z. and N.R. Mashyanov, 2000. Mercury measurements in ambient air near natural gas processing facilities. Fresenius J. Anal. Chem., 366: 429-432.

Tsuji, M., M. Hashimoto, Y. Nishizawa and T. Tsuji, 2003. Preparation of gold nanoplates by a microwave-polyol method. Chem. Lett., 32: 1114-1115. DOI:10.1246/cl.2003.1114

Tsuji, M., M. Hashimoto, Y. Nishizawa and T. Tsuji, 2004. Synthesis of gold nanorods and nanowires by microwave-polyol method. Mater. Lett., 58: 2326-2330. DOI:10.1016/j.matlet.2004.02.020

Wilhem, S.M. and N. Bloom, 2000. Mercury in petroleum. Fuel Process. Technol., 63: 1-27. DOI: 10.1016/S0378-3820(99)00068-5 